\title{
Generation of Rule-based Constraint Solvers: Combined Approach
}

\author{
Slim Abdennadher and Ingi Sobhi \\ Computer Science Department, German University in Cairo \\ [slim.abdennadher, ingi.sobhi]@guc.edu.eg \\ http://www.cs.guc.edu.eg
}

\begin{abstract}
Inductive Constraint Solving is a subfield of inductive machine learning concerned with the automatic generation of rule-based constraint solvers. In this paper, we propose an approach to generate constraint solvers given the definition of the constraints that combines the advantages of generation by construction with generation by testing. In our proposed approach, semantically valid rules are constructed symbolically, then the constructed rules are used to prune the search tree of a generate and test method. The combined approach leads in general to more expressive and efficient constraint solvers. The generated rules are implemented in the language Constraint Handling Rules.
\end{abstract}

\section{Introduction}

In rule-based constraint solving, the execution of constraints consists of a repeated application of rules. In general, we distinguish between two types of rules:

- Simplification rules that rewrite constraints to simpler constraints while preserving logical equivalence (e.g. $\min (A, A, C) \Leftrightarrow C=A$ ).

- Propagation rules that add new constraints, which are logically redundant but may cause further simplification (e.g. $\min (A, B, C) \Rightarrow C \leq A \wedge C \leq B$ ).

Writing rule-based constraint solvers is a hard task as the programmer has to determine the propagation algorithms. Several methods have been proposed in the field of inductive constraint solving to automate the generation of constraint solvers for constraints defined extensionally over finite domains by means of a truth table $[5,9,2]$ or intentionally over infinite domains by means of a constraint logic program (CLP) [3,4]. In general, the algorithms follow a generate and test approach. Rule candidates are enumerated and subjected to a validity test against the definition of the constraint.

In this paper, we present a joined approach that combines the generate and test method presented in [3] with a symbolic construction method [10]. Each method has its advantages and drawbacks. The construction method is an orthogonal approach to the general direction of the work done in the field. While it is able to generate recursive rules that cannot be generated by the generate and test 
method, it is likely to cover a narrower spectrum of rules. The generate and test method on the other hand generates a more exhaustive set of rules, however this does come at a cost. Our aim is to combine the advantages of the two approaches, while minimizing the drawbacks.

In our combined approach, we first construct semantically valid rules symbolically. Then, we use the constructed rules to prune the search tree of the generate and test method. This will generally lead to more powerful and expressive constraint solvers at a reduced cost.

In the following, we will illustrate the combined approach by an example.

Example 1. Given the following CLP program defining $\min (A, B, C)$ that holds if $C$ is the minimum of $A$ and $B$.

$$
\begin{aligned}
& \min (A, B, C) \leftarrow A \leq B \wedge C=A . \\
& \min (A, B, C) \leftarrow A>B \wedge C=B .
\end{aligned}
$$

The combined approach will construct rules symbolically.

Symbolic Construction The basic idea of the symbolic construction method stems from the observation that in general, the execution of one clause in a CLP program excludes the execution of all other clauses. Thus, to construct a simplification rule that replaces the head of a clause by the body of the clause while preserving the semantics of the CLP program, the construction algorithm adds to the head of the rule the negation of the bodies of all the other clauses. The negation of the bodies of clauses may result in a disjunction of constraints, thus for each clause a set of rules might be generated. Note that constraints that are added to the head of the rule are also added to its body to ensure that constraints removed unnecessarily are added again.

The construction algorithm generates the following simplification rules:

$$
\begin{aligned}
& \min (A, B, C) \wedge A \leq B \Leftrightarrow A \leq B \wedge C=A . \\
& \min (A, B, C) \wedge C \neq B \Leftrightarrow A \leq B \wedge C=A \wedge C \neq B . \\
& \min (A, B, C) \wedge A>B \Leftrightarrow A>B \wedge C=B . \\
& \min (A, B, C) \wedge C \neq A \Leftrightarrow A>B \wedge C=B \wedge C \neq A .
\end{aligned}
$$

To generate a simplification rule that replaces the head of the first clause with the body of the clause, the construction algorithm negates the bodies of all other clauses (i.e. second clause) to add to the head of the rule. The negation of the body of the second clause gives $A \leq B \vee C \neq B$, a disjunction of constraints. This results in two separate rules (first and second rule), one for each disjunct. Similarly, the last two rules are generated from the second clause.

Then the combined approach will eliminate the above constructed rules from the search tree of the generate and test algorithm. The generate and test algorithm used is the one proposed in [4]. 
Generate and Test All possible candidate constraints for the left hand side $C$ of the rule and right hand side $D$ of the rule are generated and tested based on the observation that a rule of the form $C \Rightarrow D$ is valid if the execution of the goal $C \wedge \neg(D)$ finitely fails with respect to the definition.

The generate and test algorithm will add the following rules to the constraint solver:

$$
\begin{aligned}
\min (A, B, C) & \Rightarrow C \leq A \wedge C \leq B . \\
\min (A, B, C) \wedge B \leq A \Leftrightarrow B \leq A & \wedge C=B .
\end{aligned}
$$

The propagation rule (first rule) is generated by calling the CLP system to execute the goal $\min (A, B, C) \wedge C>A \wedge C>B$ that fails.

The generated solvers are implemented in the language Constraint Handling Rules (CHR) [8].

The paper is organized as follows. The generate and test algorithm of [4] is summarized in Section 2. In Section 3, we present the construction algorithm. Then in Section 4, we present the combined approach. Finally, we conclude in Section 5 with a summary and future work.

\section{Generate and Test Method}

In this section, we summarize the generate and test algorithm that we use for the combined approach and which is given in [4]. The algorithm requires as input a CLP program defining the user-defined constraint for which the solver is needed.

Definition 1. A CLP program is a finite set of CLP clauses. A CLP clause is a rule of the form $H \leftarrow B_{1} \wedge \ldots \wedge B_{n} \wedge C_{1} \wedge \ldots \wedge C_{m}$ where $H, B_{1}, \ldots, B_{n}$ are atoms and $C_{1}, \ldots, C_{m}$ are built-in constraints. $H$ is called the head of the clause and $B_{1} \wedge \ldots \wedge B_{n} \wedge C_{1} \wedge \ldots \wedge C_{m}$ is called the body of the clause. A user-defined constraint is defined in a CLP program if it occurs in the head of the clause.

The algorithm also requires the following sets which specify the syntactic form of the generated rules of the solver:

- A set of built-in and user-defined constraints denoted by Baselhs. These constraints are the common part that must appear in the left hand side (lhs) of all rules.

- A set of built-in and user-defined constraints denoted by $C_{a n d}$ lhs. These are the candidate constraints to be used in conjunction with the Base lhs to form the lhs of a rule.

- A set containing built-in constraints denoted by Cand $_{r h s}$. These are the candidate constraints that may appear in the right hand side (rhs) of a rule. This set can be expanded to contain user-defined constraints. 
Example 2. To generate a constraint solver for min constraint of Example 1, the algorithm takes as input the CLP program defining the constraint, as well as, the following sets:

$$
\begin{aligned}
\text { Base }_{l h s}= & \{\min (A, B, C)\} \\
\text { Cand }_{l h s}= & \{A=B, A=C, B=C, A \neq B, A \neq C, B \neq C, \\
& A \leq B, A \leq C, B \leq A, B \leq C, C \leq A, C \leq B\} \\
\text { Cand }_{\text {rhs }}= & \text { Cand }_{\text {lhs }}
\end{aligned}
$$

Example 3. To generate a constraint solver for append $(A, B, C)$ that holds if list $C$ is the concatenation of lists $A$ and $B$, the algorithm takes as input the following CLP program:

$$
\begin{aligned}
& \operatorname{append}(A, B, C) \leftarrow A=[] \wedge C=B . \\
& \operatorname{append}(A, B, C) \leftarrow A=[D \mid E] \wedge C=[F \mid G] \wedge D=F \wedge \operatorname{append}(E, B, G) .
\end{aligned}
$$

As well as, the following sets:

$$
\begin{aligned}
\text { Base }_{l h s}= & \left\{\text { append }_{(A, B, C)\}}\right. \\
\text { Cand }_{l h s}= & \{A=[], B=[], C=[], A=B, A=C, B=C, A \neq B, A \neq C, B \neq C, \\
& A \neq[], B \neq[], C \neq[]\} \\
\text { Cand }_{r h s}= & \text { Cand }_{l h s}
\end{aligned}
$$

Given the specified input parameters, candidate propagation rules are generated of the form $C \Rightarrow D$, where $C$ the lhs of the rule is a subset of $B_{a s e} e_{l h s} \cup C a n d_{l h s}$, and $D$ the rhs of the rule is a subset of $\operatorname{Cand}_{r h s}$. The candidate rules are then subjected to a validity test as follows:

- For primitive propagation rules (i.e. rules with rhs consisting of only built-in constraints), the validity test is based on the observation that a rule of the form $C \Rightarrow D$ is valid if the execution of the goal $C \wedge \neg(D)$ finitely fails with respect to the given CLP program and the predefined solver for the built-in constraints.

- For general propagation rules (i.e. rules with rhs consisting of both built-in and user-defined constraints) to avoid the problems relating to the negation of user-defined constraints, a different validity test is proposed where the negation is performed on the set of answers to a goal (set of constraints) rather than on the constraints themselves.

For the execution of the goals, a bounded depth tabled resolution [6,7] for CLP is used to avoid non-termination. The intuitive basic principle of tabled resolution is the following: each new subgoal $S$ is compared to the previous intermediate subgoals (not necessarily in the same branch of the resolution tree). If there is a previous subgoal $I$ which is equivalent to $S$ or more general than $S$, then no more unfolding is performed on $S$ and answers for $S$ are selected among the answers of $I$. This process is repeated for all subsequent computed answers that correspond to the subgoal $I$. 
Example 4. Consider the following primitive propagation rule which is generated by the algorithm for the append constraint:

$$
\operatorname{append}(A, B, C) \wedge B=[] \Rightarrow A=C \text {. }
$$

The validity test for the rule is determined from the execution of the goal append $(A, B, C) \wedge B=[] \wedge A \neq C$. Using a classical CLP resolution scheme, the goal will lead to an infinite derivation tree, whereas in the case of a tabled resolution, the execution of the goal will fail as shown by the derivation tree below:

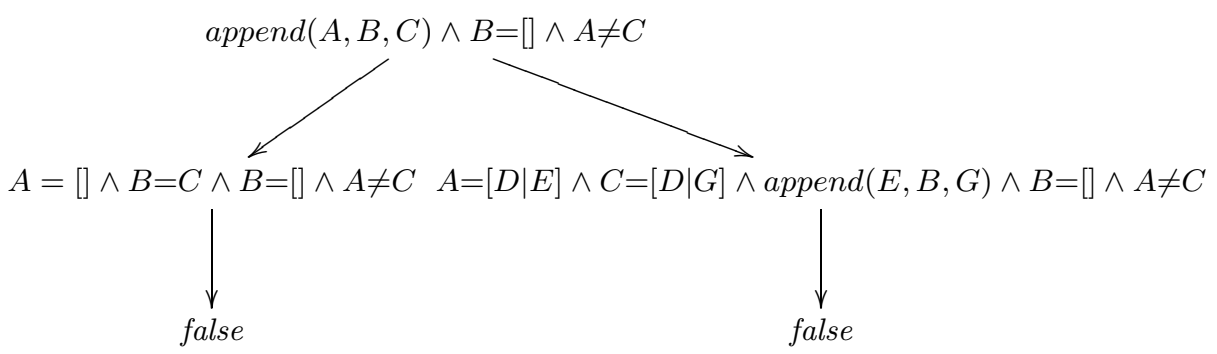

The initial goal $G_{1}=(\operatorname{append}(A, B, C) \wedge B=[] \wedge A \neq C)$ is more general than the subgoal $G_{2}=(A=[D \mid E] \wedge C=[D \mid G] \wedge \operatorname{append}(E, B, G) \wedge B=[] \wedge A \neq C)$, in the sense that $(\operatorname{append}(X, Y, Z) \wedge U=[W \mid X] \wedge V=[W \mid Z] \wedge Y=[] \wedge U \neq V)$ entails $(\operatorname{append}(X, Y, Z) \wedge Y=[] \wedge X \neq Z)$. So no unfolding is made on $G_{2}$, and the process waits for answers of $G_{1}$ to compute answers of $G_{2}$. Since $G_{1}$ has no further possibility of having answers, then $G_{2}$ fails and thus $G_{1}$ also fails.

Since a propagation rule does not remove constraints but adds new ones, the constraint store may contain superfluous information. To improve the time and space behavior of constraint solving, propagation rules should be transformed into equivalent simplification rules. For some of the automatically generated propagation rules a transformation to simplification rules is possible. For a valid propagation rule of the form $C \Rightarrow D$, if a proper subset $E$ of $C$ can be found such that $D \cup E \Rightarrow C$ is valid too then the propagation rule can be transformed to a simplification rule of the form $C \Leftrightarrow D \cup E$.

Example 5. For the min constraint of Example 1, the generate and test algorithm generates the following valid rules:

$$
\begin{array}{r}
\min (A, B, C) \Rightarrow C \leq A \wedge C \leq B . \\
\min (A, B, C) \wedge C \neq A \Leftrightarrow C=B \wedge C \neq A . \\
\min (A, B, C) \wedge C \neq B \Leftrightarrow C=A \wedge C \neq B . \\
\min (A, B, C) \wedge A \leq B \Leftrightarrow C=A \wedge A \leq B . \\
\min (A, B, C) \wedge B \leq A \Leftrightarrow C=B \wedge B \leq A .
\end{array}
$$

The set of generated rules is complete, i.e. it propagates all built-in constraints (equalities and inequalities) that logically follow from the min constraint definition and some given equalities or inequalities. 
Example 6. For the append constraint of Example 3, the generate and test algorithm generates among others the following valid rules:

$$
\begin{aligned}
& \operatorname{append}(A, B, C) \wedge A=[] \Leftrightarrow A=[] \wedge B=C . \\
& \operatorname{append}(A, B, C) \wedge B=[] \Leftrightarrow A=C \wedge B=[] . \\
& \operatorname{append}(A, B, C) \wedge C=[] \Leftrightarrow A=[] \wedge B=[] \wedge C=[] . \\
& \operatorname{append}(A, B, C) \wedge A=C \Leftrightarrow B=[] \wedge A=C . \\
& \operatorname{append}(A, B, C) \wedge A \neq[] \Rightarrow C \neq[] . \\
& \operatorname{append}(A, B, C) \wedge B \neq[] \Rightarrow A \neq C \wedge C \neq[] .
\end{aligned}
$$

The rules handle only special cases, where equality or inequality constraints are checked between the arguments of the constraint and the empty list. The solver is incomplete due to the absence of recursive rules that are able to handle more general cases.

\section{Symbolic Construction Method}

In this section, we present an algorithm that constructs simplification rules symbolically for a constraint $H$ defined by a CLP program, as follows:

$$
H \leftarrow C_{1}, H \leftarrow C_{2}, \ldots, H \leftarrow C_{n} .
$$

where $C_{i}$ is a conjunction of constraints, $n$ is the total number of clauses and the clauses are non-overlapping (i.e. in a computation at most one clause can be chosen for a goal). Note that any overlapping CLP program can be transformed into an equivalent non-overlapping one.

The algorithm is presented in Figure 1. The basic idea of the algorithm stems from the observation that in general, the execution of one clause in a CLP program excludes the execution of all other clauses. Thus, to construct a valid simplification rule that simplifies the constraint $H$ to $C_{i}$ (the body of the $i t h$ clause), the negation of the bodies of all other clauses is added to the head of the rule to ensure that the rule will only be applicable if the bodies of all the other clauses are not. This is needed to preserve the semantics of the CLP program defining the constraint.

The algorithm works as follows. For each clause $H \leftarrow C_{i}$ in the CLP program, it constructs the simplification rule(s) by:

- Setting the head of the rule to $H$.

- Setting the body of the rule to $C_{i}$.

- Adding to the head of the rule $G_{i}^{j}$; a disjunct from $G_{i}$, the expression resulting from negating the bodies of all the CLP clauses excluding $C_{i}$.

- Adding to the body of the rule $G_{i}^{j}$. This is done to ensure that constraints removed unnecessarily from the constraint store are added again.

The constructed simplification rules are of the form:

$$
H \wedge G_{i}^{j} \Leftrightarrow C_{i} \wedge G_{i}^{j} \quad 1 \leq j \leq m_{i}, 1 \leq i \leq n
$$

where $G_{i}^{j}$ is a conjunction of built-in constraints from $G_{i}$ and $m_{i}$ is the number of disjuncts $G_{i}^{j}$ in $G_{i}$. 
begin

$H$ : the head of the clauses.

$B$ : the set of clause bodies.

$R$ : the set of resultant simplification rules initialized to [].

while $B$ is not empty do

Remove from $B$ its first element denoted $C_{i}$.

Other $_{B}$ : the set of all clause bodies except $C_{i}$.

$G_{i}$ : the set resulting from negating Other $_{B}$.

while $G_{i}$ is not empty do

Remove from $G_{i}$ its first element denoted $G_{i}^{j}$.

Add rule $\left(H \wedge G_{i}^{j} \Leftrightarrow C_{i} \wedge G_{i}^{j}\right)$ to $R$.

end while

end while

end

Fig. 1. The Symbolic Construction Algorithm

Determination of $\boldsymbol{G}_{\boldsymbol{i}}$ Given a clause $H \leftarrow C_{i}$, the expression $G_{i}$ is formally determined as follows:

- Negate the bodies of all clauses of the CLP program except the body $C_{i}$ :

$$
\neg\left(C_{1} \vee \ldots \vee C_{i-1} \vee C_{i+1} \vee \ldots \vee C_{n}\right)
$$

- Distribute the negation:

$$
\neg C_{1} \wedge \ldots \wedge \neg C_{i-1} \wedge \neg C_{i+1} \wedge \ldots \wedge \neg C_{n}
$$

Since $C_{i}$ is a conjunction of constraints, this expands to:

$$
\begin{aligned}
& \neg\left(c_{1}^{1} \wedge \ldots \wedge c_{1}^{k_{1}}\right) \wedge \ldots \wedge \neg\left(c_{(i-1)}^{1} \wedge \ldots \wedge c_{(i-1)}^{k_{(i-1)}}\right) \wedge \\
& \neg\left(c_{(i+1)}^{1} \wedge \ldots \wedge c_{(i+1)}^{k_{(i+1)}}\right) \wedge \ldots \wedge \neg\left(c_{n}^{1} \wedge \ldots \wedge c_{n}^{k_{n}}\right)
\end{aligned}
$$

where $k_{i}$ denotes the number of constraints in a body $C_{i}$.

- Push the negation into the conjunctions. This transforms the conjunctions of constraints to disjunctions of negated constraints:

$$
\begin{aligned}
& \left(\neg c_{1}^{1} \vee \ldots \vee \neg c_{1}^{k_{1}}\right) \wedge \ldots \wedge\left(\neg c_{(i-1)}^{1} \vee \ldots \vee \neg c_{(i-1)}^{k_{(i-1)}}\right) \wedge \\
& \left(\neg c_{(i+1)}^{1} \vee \ldots \vee \neg c_{(i+1)}^{k_{(i+1)}}\right) \wedge \ldots \wedge\left(\neg c_{n}^{1} \vee \ldots \vee \neg c_{n}^{k_{n}}\right)
\end{aligned}
$$

- Replace each negated constraint $\neg c_{q}^{d}$ by a corresponding simplified positive constraint. The algorithm distinguishes between two cases: 
- If $c_{q}^{d}$ is a built-in constraint, the algorithm replaces $\neg c_{q}^{d}$ by its corresponding positive constraint after simplification. The set of built-in constraints is assumed to be closed under negation. For obtained constraints that consist of local variables (i.e. variables that do not occur in $H$ ), the algorithm adds the built-in constraints (in their positive form) from the body $C_{q}$ that define the local variables.

- Otherwise, $c_{q}^{d}$ is a user-defined constraint and since the negation of userdefined constraints is still not well-defined, the algorithm discards $\neg c_{q}^{d}$ (i.e. no rules will be constructed for this case).

This results in a formula of the form:

$$
\begin{aligned}
& \left(P_{1}^{1} \vee \ldots \vee P_{1}^{l_{1}}\right) \wedge \ldots \wedge\left(P_{(i-1)}^{1} \vee \ldots \vee P_{(i-1)}^{l_{(i-1)}}\right) \wedge \\
& \left(P_{(i+1)}^{1} \vee \ldots \vee P_{(i+1)}^{l_{(i+1)}}\right) \wedge \ldots \wedge\left(P_{n}^{1} \vee \ldots \vee P_{n}^{l_{n}}\right)
\end{aligned}
$$

where $P_{i}^{e}$ is a built-in constraint or a conjunction of built-in constraints and $l_{i}$ denotes the number of built-in constraints in a disjunct $C_{i}$.

- Distribute the conjunction over the disjunction:

$$
\begin{aligned}
& \left(P_{1}^{1} \wedge \ldots \wedge P_{(i-1)}^{1} \wedge P_{(i+1)}^{1} \wedge \ldots \wedge P_{n}^{1}\right) \vee \ldots \vee \\
& \left(P_{1}^{l_{1}} \wedge \ldots \wedge P_{(i-1)}^{l_{(i-1)}} \wedge P_{(i+1)}^{l_{(i+1)}} \wedge \ldots \wedge P_{n}^{l_{n}}\right)
\end{aligned}
$$

This results in $G_{i}$, which is a formula in disjunctive normal form $G_{i}^{1} \vee \ldots \vee$ $G_{i}^{m_{i}}$, where $G_{i}^{j}$ is a conjunction of built-in constraints.

Example 7. Given the CLP program for the append of Example 3:

$$
\begin{aligned}
& \operatorname{append}(A, B, C) \leftarrow A=[] \wedge C=B . \\
& \operatorname{append}(A, B, C) \leftarrow A=[D \mid E] \wedge C=[F \mid G] \wedge D=F \wedge \operatorname{append}(E, B, G) .
\end{aligned}
$$

The symbolic construction algorithm will construct rules for the first clause by setting the head of the rules to append $(A, B, C)$ and the body of the rules to the body of the clause, $A=[] \wedge C=B$. It then determines $G_{1}$, the expression resulting from negating the bodies of all other clauses as follows:

- Negate the body of the second clause:

$$
\neg(A=[D \mid E] \wedge C=[F \mid G] \wedge D=F \wedge \operatorname{append}(E, B, G))
$$

- Distribute the negation:

$$
\neg(A=[D \mid E]) \vee \neg(C=[F \mid G]) \vee \neg(D=F) \vee \neg(\operatorname{append}(E, B, G))
$$

- Given that the equality constraint is a built-in constraint defined by a constraint theory and for which a solver is available, the algorithm performs the following operations: 
- It replaces $\neg(A=[D \mid E])$ and $\neg(C=[F \mid G])$ by $A \neq[D \mid E]$ and $C \neq[F \mid G]$ which will be simplified by the built-in solver to $A=[]$ and $C=[]$, respectively.

- It replaces $\neg(D=F)$ by $D \neq F$. Since $D$ and $F$ are local variables, the built-in constraints $A=[D \mid E]$ and $C=[F \mid G]$ that define the local variables to be the first elements of the lists $A$ and $C$ are added.

- Negated user-defined constraint $\neg(\operatorname{append}(E, B, G))$ is discarded.

This results in

$$
A=[] \vee C=[] \vee(D \neq F \wedge A=[D \mid E] \wedge C=[F \mid G])
$$

and the following three simplification rules are constructed:

$$
\begin{aligned}
& \operatorname{append}(A, B, C) \wedge A=[] \Leftrightarrow A=[] \wedge C=B . \\
& \operatorname{append}(A, B, C) \wedge C=[] \Leftrightarrow A=[] \wedge C=B \wedge C=[] . \\
& \operatorname{append}(A, B, C) \wedge D \neq F \wedge A=[D \mid E] \wedge C=[F \mid G] \Leftrightarrow A=[] \wedge C=B \wedge \\
& \qquad D \neq F \wedge A=[D \mid E] \wedge C=[F \mid G] .
\end{aligned}
$$

Similarly, the following simplification rules are constructed for the second clause:

$$
\begin{aligned}
\operatorname{append}(A, B, C) \wedge A \neq[] \Leftrightarrow A= & {[D \mid E] \wedge C=[F \mid G] \wedge D=F \wedge } \\
& \quad \operatorname{append}(E, B, G) \wedge A \neq[] . \\
\operatorname{append}(A, B, C) \wedge C \neq B \Leftrightarrow A= & {[D \mid E] \wedge C=[F \mid G] \wedge D=F \wedge } \\
& \quad \operatorname{append}(E, B, G) \wedge C \neq B .
\end{aligned}
$$

The rules are recursive. The power of the symbolic construction algorithm is in the generation of such recursive rules given a recursive constraint definition.

Simplification In general, the simplification rules constructed are not in the simplest form. To simplify the constructed rules, the head and body of the rules are executed against the solvers for the built-in constraints.

Example 8. Consider the following constructed rule for append:

$$
\begin{gathered}
\operatorname{append}(A, B, C) \wedge D \neq F \wedge A=[D \mid E] \wedge C=[F \mid G] \Leftrightarrow \underline{A=[]} \wedge C=B \wedge \\
D \neq F \wedge \underline{A=[D \mid E]} \wedge C=[F \mid G] .
\end{gathered}
$$

Since the existence of the constraints $A=[]$ and $A=[D \mid E]$ leads to a contradiction, the rule will be simplified to :

$$
\operatorname{append}(A, B, C) \wedge D \neq F \wedge A=[D \mid E] \wedge C=[F \mid G] \Leftrightarrow \text { false. }
$$


Redundancy In general, the generated rules may contain redundant rules. To remove redundant rules, the same algorithm is used as the one summarized in the redundancy pruning in Section 4, which basically states that a rule is redundant and should be removed if its operation is covered by the remaining rules of the solver.

Example 9. Consider the following two rules of the constructed solver for append:

$$
\begin{gathered}
\operatorname{append}(A, B, C) \wedge D \neq F \wedge A=[D \mid E] \wedge C=[F \mid G] \Leftrightarrow \text { false. } \\
\operatorname{append}(A, B, C) \wedge A \neq[] \Leftrightarrow A=[D \mid E] \wedge C=[F \mid G] \wedge D=F \wedge \\
\quad \operatorname{append}(E, B, G) .
\end{gathered}
$$

The first rule is redundant and can be removed since removing it and executing the goal append $(A, B, C) \wedge D \neq F \wedge A=[D \mid E] \wedge C=[F \mid G]$ on the remaining rules, the second rule will be fired and leads to a contradiction.

Example 10. For the append of Example 3, the simplification rules reduce to the following set:

$$
\begin{aligned}
\operatorname{append}(A, B, C) \wedge A=[] \Leftrightarrow C=B \wedge A=[] . & \\
\operatorname{append}(A, B, C) \wedge C=[] \Leftrightarrow C=B \wedge A=[] \wedge C=[] . & \\
\operatorname{append}(A, B, C) \wedge A \neq[] \Leftrightarrow A=[D \mid E] \wedge C=[F \mid G] \wedge D=F \wedge & \operatorname{append}(E, B, G) . \\
\operatorname{append}(A, B, C) \wedge C \neq B \Leftrightarrow A=[D \mid E] \wedge & C=[F \mid G] \wedge D=F \wedge \\
& \quad \operatorname{append}(E, B, G) \wedge C \neq B .
\end{aligned}
$$

The rules cover some of the cases, where list $A$ is empty (first and second rules), as well as, when it consists of at least one element (third and fourth rules). In the latter case, the simplification rule is called recursively on each of the elements of list $A$. However, it should be noted that the solver is not propagation complete, i.e. it does not produce all built-in constraints that logically follows from the constraint definition such as that the list $B$ is empty if it is known that the lists $A$ and $C$ are equal.

Recursive Rules The power of the symbolic construction approach is its ability to generate recursive rules which cannot be generated by other approaches.

Example 11. Consider the following CLP program that defines the constraint replace $(A, B, C, D)$ that holds if list $D$ is the result of replacing all occurrences of $A$ in list $C$ by $B$.

$$
\operatorname{replace}(A, B, C, D) \leftarrow C=[] \wedge D=[] .
$$




$$
\begin{aligned}
\operatorname{replace}(A, B, C, D) \leftarrow C=[E \mid F] \wedge D=[G \mid H] \wedge & E=A \wedge G=B \wedge \\
& \operatorname{replace}(A, B, F, H) . \\
\operatorname{replace}(A, B, C, D) \leftarrow C=[E \mid F] \wedge D=[G \mid H] \wedge & E \neq A \wedge G=E \wedge \\
& \operatorname{replace}(A, B, F, H) .
\end{aligned}
$$

The symbolic construction algorithm will generate the following simplification rules:

$$
\begin{aligned}
& \operatorname{replace}(A, B, C, D) \wedge C=[] \Leftrightarrow C=[] \wedge D=[] . \\
& \operatorname{replace}(A, B, C, D) \wedge D=[] \Leftrightarrow C=[] \wedge D=[] . \\
& \operatorname{replace}(A, B, C, D) \wedge C=[E \mid F] \wedge E=A \Leftrightarrow C=[E \mid F] \wedge D=[G \mid H] \wedge \\
& \qquad E=A \wedge G=B \wedge \operatorname{replace}(A, B, F, H) . \\
& \operatorname{replace}(A, B, C, D) \wedge C=[E \mid F] \wedge E \neq A \Leftrightarrow C=[E \mid F] \wedge D=[G \mid H] \wedge \\
& \operatorname{replace}(A, B, C, D) \wedge D=[G \mid H] \wedge G \neq B \Leftrightarrow C=[E \mid F] \wedge D=[G \mid H] \wedge \\
& \operatorname{replace}(A, B, C, D) \wedge C=[E \mid F] \wedge D=[G \mid H] \wedge G \neq E \Leftrightarrow C=[E \mid F] \wedge \\
& \qquad D=[G \mid H] \wedge E=A \wedge G=B \wedge G \neq E \wedge \operatorname{replace}(A, B, F, H) .
\end{aligned}
$$

The symbolic construction algorithm constructs the rules by direct derivation from the definition. The first two rules apply when the lists are empty. The last four rules apply when information is known about either of the leading list elements $E$ or $G$ or when the relationship between them is sufficiently known. The rules do not cover all possibilities, however they represent a good basis for a constraint solver for replace $(A, B, C, D)$.

\section{Combined Approach}

Both the symbolic construction method and the generate and test method have advantages and disadvantages. The symbolic construction method is able to generate recursive rules where all other approaches based on generation and testing failed. However, the generate and test, in general, generates a more exhaustive set of rules.

In this section, we will present a combination of the symbolic construction method and the generate and test method that will lead to more powerful and expressive constraint solvers at a reduced cost of generation.

We will first construct semantically valid rules using the symbolic construction method then we will use the generated rules to prune the search tree of the generate and test method using the closure pruning technique. However, even with this pruning technique, the combined approach generates redundant rules that should be removed. This will be done using the second pruning technique. 
1. Closure Pruning: If a rule of the form $C \Leftrightarrow D$ is generated using the symbolic construction algorithm then in the generate and test method there is no need to consider rules where the lhs constraint is $C$. Thus, during the enumeration of all possible rule lhs, unnecessary lhs candidates are removed from this list. For efficiency reasons, the concrete implementation is not based on a list but on a tree containing lhs candidates on its nodes.

2. Redundancy Pruning: To suppress the generation of redundant rules, we use the algorithm proposed in [1]. The idea of the algorithm is based on operational equivalence of programs. The operational equivalence test for redundancy removal is to check if the computation step due to the candidate rule that is tested for redundancy can be performed by the remainder of the program. This is done by executing the prefix of the candidate rule in both programs and comparing the results. If the results are identical, then the rule is obviously redundant and can be removed.

A redundant rule is defined formally as follows:

Definition 2. A rule $R$ is redundant in a program $P$ if and only if for all states $S:$ If $S \mapsto_{P}^{*} S_{1}$ then $S \mapsto_{P}^{*} \backslash\{R\} S_{2}$, where $S_{1}$ and $S_{2}$ are final states and are identical upto renaming of variables and logical equivalence of builtin constraints. $\mapsto_{P}^{*}$ denotes the reflexive and transitive closure of $\mapsto_{P}$.

The redundancy pruning technique is non-deterministic since the resulting solver may vary depending on the order in which rules are tried and removed.

Example 12. For the min constraint of Example 1, the symbolic construction method generates the following rules:

$$
\begin{aligned}
& \min (A, B, C) \wedge A \leq B \Leftrightarrow A \leq B \wedge C=A . \\
& \min (A, B, C) \wedge C \neq B \Leftrightarrow A \leq B \wedge C=A \wedge C \neq B . \\
& \min (A, B, C) \wedge A>B \Leftrightarrow A>B \wedge C=B . \\
& \min (A, B, C) \wedge C \neq A \Leftrightarrow A>B \wedge C=B \wedge C \neq A .
\end{aligned}
$$

The generate and test algorithm will first generate the propagation rule (Rule 1). Using the closure pruning technique, Rules 2,3 , and 4 are not checked. Rule 5 will be generated since there is no rule that checks for $B \leq A$. Combining both sets of rules, Rule 8 will be eliminated using the redundancy pruning technique since it is covered by Rule 5 . The combined approach generates the same rules as the ones generated using the generate and test method however less candidate rules are checked.

In general, the set of rules generated using the combined approach is more expressive and powerful than the ones generated either using the generate and test method or using the symbolic construction method as illustrated in the following example.

Example 13. For the append constraint, the combined approach generates the following rules using the symbolic construction method:

$$
\operatorname{append}(A, B, C) \wedge A=[] \Leftrightarrow C=B \wedge A=[] \text {. }
$$




$$
\begin{aligned}
& \operatorname{append}(A, B, C) \wedge C=[] \Leftrightarrow C=B \wedge A=[] \wedge C=[] . \\
& \operatorname{append}(A, B, C) \wedge A \neq[] \Leftrightarrow A=[D \mid E] \wedge C= {[F \mid G] \wedge D=F \wedge } \\
& \quad \operatorname{append}(E, B, G) . \\
& \operatorname{append}(A, B, C) \wedge C \neq B \Leftrightarrow A=[D \mid E] \wedge C=[F \mid G] \wedge D=F \wedge \\
& \quad \operatorname{append}(E, B, G) \wedge C \neq B .
\end{aligned}
$$

Then the following rules (among others) will be added from the generate and test method:

$$
\begin{aligned}
& \operatorname{append}(A, B, C) \wedge B=[] \Leftrightarrow A=C \wedge B=[] . \\
& \operatorname{append}(A, B, C) \wedge A=C \Leftrightarrow B=[] \wedge A=C . \\
& \operatorname{append}(A, B, C) \wedge B \neq[] \Rightarrow A \neq C .
\end{aligned}
$$

Adding these rules improves the efficiency of the solver. For example, with Rule 10 the recursion over the list $\mathrm{A}$ is replaced by a simple unification $A=C$ if list $B$ is empty.

Implementation in CHR The head of the generated rules may contain constraints that are built-in constraints for the CHR system. To have a running CHR solver, these constraints should be removed from the head. This is done in two steps:

- Equality constraints appearing in the head of a rule are propagated all over the constraints in the head and body of the rule. Then the resulting constraints are simplified. This can be performed as follows. In turn, each equality constraint appearing in the head is removed and transformed in a substitution that is applied to the head and body.

- For other built-in constraints, the transformation leads to guarded CHR rules [8].

Example 14. The following simplification rule for min:

$$
\min (A, B, C) \wedge B \leq A \Leftrightarrow B \leq A \wedge C=B
$$

will be transformed to the following guarded CHR simplification rule:

$$
\min (A, B, C) \Leftrightarrow B \leq A \mid C=B .
$$

Equivalent Definitions - Same Solvers The generate and test method is based on enumerating rule candidates and checking their validity against the intentional definition. Thus, having two equivalent definitions the generate and test will generate always the same set of rules.

However, using the symbolic construction method, the set of generated rules for a constraint may differ for different but equivalent definitions of the constraint. 
The following example will show that the more compact the set of clauses is, the more expressive the constructed solver is. This is intuitively clear since the construction method generates rules for a clause by negating the bodies of all other clauses which are added to the head and the body of the rule. In general, negating more than a clause will lead to adding more than one constraint to the head of the rule making it more restrictive.

Example 15. The constraint min of Example 1 can be defined by an equivalent CLP program consisting of three clauses instead of two as follows:

$$
\begin{aligned}
& \min (A, B, C) \leftarrow A<B \wedge C=A . \\
& \min (A, B, C) \leftarrow A>B \wedge C=B . \\
& \min (A, B, C) \leftarrow A=B \wedge C=A .
\end{aligned}
$$

The symbolic construction algorithm generates the following set of simplification rules:

$$
\begin{aligned}
& \min (A, B, C) \wedge A<B \Leftrightarrow C=A \wedge A<B . \\
& \min (A, B, C) \wedge A>B \Leftrightarrow C=B \wedge A>B . \\
& \min (A, B, C) \wedge A=B \Leftrightarrow C=A \wedge C=B \wedge A=B . \\
& \min (A, B, C) \wedge C \neq A \Leftrightarrow C=B \wedge A>B \wedge C \neq A . \\
& \min (A, B, C) \wedge C \neq B \wedge A \neq B \Leftrightarrow C=A \wedge A<B \wedge C \neq B . \\
& \min (A, B, C) \wedge C \neq B \wedge A \geq B \Leftrightarrow \text { false. }
\end{aligned}
$$

Although the number of generated rules has increased compared to the set of rules presented in Example 1, these rules are less expressive since:

- Rule 6 subsumes the two rules 13 and 15. Whereas Rule 6 will be applied for the goal $\min (A, B, C) \wedge A \leq B$, no rule is applicable using the rules above.

- Rule 7 of the first solver is more general than its counterparts, Rule 17 and Rule 18.

Using the combined approach, all rules of the generate and test method will be added except Rule 16 which will not be checked or generated. Using the redundancy pruning technique, all rules of the construction method will be removed except Rule 16. The resulting solver of the combined approach is identical to the solver generated for the min constraint defined using two clauses. However, it should be noted that the solver obtained by construction using only two clauses pruned the search tree better.

\section{Conclusion}

In this paper, we have extended the work done in the field of Inductive Constraint Solving by providing a method that combines the advantages of the generate and test approach with a symbolic construction method based on rewriting of CLP programs. 
In the combined approach, we first generate rules using the symbolic construction method then we use them to prune the search tree of the generate and test method. In general, the combined approach leads to more expressive and efficient constraint solvers at a reduced cost. Some rules, like recursive rules that cannot be generated using the generate and test method are generated using the symbolic construction method.

One interesting direction for future work is to investigate the completeness of the solvers generated. It is clear that in general this property cannot be guaranteed, but in some cases it should be possible to check it, or at least to characterize the kind of consistency the solver can ensure.

\section{References}

1. S. Abdennadher and T. Frühwirth. Integration and Optimization of Rule-based Constraint Solvers. In International Symposium on Logic-based Program Synthesis and Transformation, LOPSTR03, LNCS. Springer, 2004.

2. S. Abdennadher and C. Rigotti. Automatic Generation of Propagation Rules for Finite Domains. In 6th International Conference on Principles and Practice of Constraint Programming, CP00, LNCS 1894. Springer-Verlag, 2000.

3. S. Abdennadher and C. Rigotti. Towards Inductive Constraint Solving. In 7th International Conference on Principles and Practice of Constraint Programming, CP01, LNCS 2239, pages 31-45. Springer-Verlag, 2001.

4. S. Abdennadher and C. Rigotti. Automatic Generation of CHR Constraint Solvers. Journal of Theory and Practice of Logic Programming (TPLP), 5(2), 2005.

5. K. Apt and E. Monfroy. Automatic Generation of Constraint Propagation Algorithms for Small Finite Domains. In 5th International Conference on Principles and Practice of Constraint Programming, CP99, LNCS 1713. Springer-Verlag, 1999.

6. P. Codognet. A Tabulation Method for Constraint Logic Programs. In 8th Symposuim and Exibition on Industrial Applications of Prolog, 1995.

7. B. Cui and D. S. Warren. A System for Tabled Constraint Logic Programming. In 1st International Conference on Computational Logic, LNCS 1861. SpringerVerlag, 2000.

8. T. Frühwirth. Theory and Practice of Constraint Handling Rules, Special Issue on Constraint Logic Programming. Journal of Logic Programming, 37(1-3):95-138, 1998.

9. C. Ringeissen and E. Monfroy. Generating Propagation Rules for Finite Domains: A Mixed Approach. In New Trends in Constraints, LNAI 1865, pages 150-172, 2000 .

10. I. Sobhi. Constructive generation of rule-based constraint solvers. Master's thesis, German University in Cairo, 2007. 